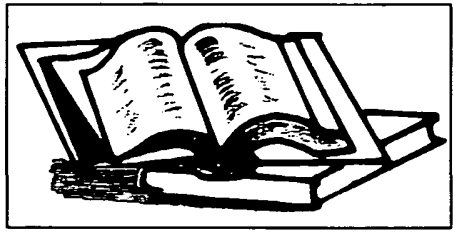

Sources Statistiques sur l'emploi dans le secteur public

ORGANISATION DE COOPERATION ET DE DEVELOPPEMENT ECONOMIQUES (O.C.D.E.). París, 1994. 183 páginas.

\section{I) Introducción}

La presente obra, también publicada en inglés con el título Statistical Sources on Public Sector Employment, es ante todo el resultado de una amplia investigación realizada conjuntamente por la Oficina Internacional del Trabajo (O.I.T.) y la O.C.D.E. acerca de las fuentes de datos estadísticos sobre el empleo en el sector público en los distintos países de la O.C.D.E.

El estudio a su vez se compone de dos grandes apartados, que comprenden distintos capítulos o subapartados, elaborados respectivamente por Bernard FEYS del Servicio de Gestión Pública de la O.C.D.E. y por Magali IMBERT-LUCCIONI del Departamento de Estadística de la Oficina Internacional del Trabajo; relativos, el primero, a una propuesta de análisis internacional comparado de las estadísticas de empleo público en los países miembros de la O.C.D.E., y el segundo, a un análisis descriptivo y detallado de las distintas fuentes de datos nacionales existentes.

\section{II) La comparación internacional de las estadísticas de empleo público en los países miembros de la O.C.D.E.}

Este primer gran apartado de la obra que se glosa, refleja los esfuerzos realizados en orden a la posibilidad de hacer comparaciones entre los sectores públicos de los distintos países miembros de la O.C.D.E., a partir de la existencia de datos estadísticos normalizados y fiables.

La finalidad de la comparación de las estadísticas nacionales sobre el empleo en el sector público sería no sólo el conocimiento de esta importante fuente de información, sino también y sobre todo el permitir a medio y largo plazo hacer propuestas e iniciativas de innovación administrativa desde una perspectiva internacional, mucho más amplia. Ahora bien, lo anterior choca de entrada con un gran obstáculo, que es nada más y nada menos que para poder comparar las estadísticas nacionales sobre el empleo en el sector público en particular y en general sobre la gestión pública en los distintos países de la O.C.D.E., hace falta previamente que las definiciones sobre lo que se entiende por sector público en cada país puedan ser comparables.

Con la intención de lograr esa antedicha comparación, se parte de los resultados obtenidos a partir de la información recibida de veintidós países de la O.C.D.E. de una encuesta que les fue enviada en la primavera de 1992, completados con las visitas realizadas a esos países con el fin de analizar en mayor profundidad o aclarar los datos recibidos.

Las principales conclusiones obtenidas de la información estadística remitida por los distintos páses, fueron las siguientes: a) en ocasiones existe descoordinación en las instituciones que han elaborado y enviado las estadísticas, debido a que en el seno de las mismas existen a veces varias fuentes o bancos de datos; b) no se utilizan con un carácter estricto las definiciones y clasificaciones internacionales normalizadas y más comunes acerca del sector público y las distintas instituciones que lo componen en cada país; c) existen países donde la información estadística se elabora por varias instituciones, con técnicas y denominaciones a veces muy imprecisas y confusas, y descoordinadas entre sí; d) en particular, los conceptos de empresas públicas y servicios públicos son difíciles de comparar entre países por sus notables diferencias de definición y de contenidos.

A fin de evitar las importantes dificultades e insuficiencias observadas en la posibilidad de comparación de los sectores públicos de los distintos países de la O.C.D.E. y de poder avanzar en ésta, se proponen básicamente dos vías de actuación, las cuales pienso que son de indudable interés y utilidad, a saber: a) En primer lugar, el establecimiento de un sistema de estadísticas comparado, tarea que implicará notables esfuerzos y en la que se ya ha comenzado desde hace unos cuantos años mediante un Grupo de Trabajo creado por las Naciones Unidas, y en el que participan la O.C.D.E., el Fondo Monetario Internacional, el Banco Mundial y E.U.R.O.S.T.A.T. (Oficina de Estadística de las Comunidades Europeas); b) en segundo término, el intentar que se comparen preferentemente series cronológicas, antes que estadísticas o datos meramente transversales, siendo para ello necesaria la permanente estabilidad de las definiciones prefijadas sobre el sector público.

Como resultado de la actividad del Grupo de Trabajo antedicho, existe en la actualidad un Breve Informe-Resumen que, data de 1993, sobre la revisión aprobada del Sistema de Contabilidad Nacional, que tiene, como es sabido, su origen en 1968, en la que en absoluto se modifican las definiciones precedentes sobre el sector público, pero que, por el contrario, aporta una importante clasificación para su interpretación y delimitación. Ello se realiza a través de la fijación de dos criterios básicos, relativos a la definición de Administraciones Públicas: a) el criterio no mercantil de su actuación, y b) el grado de financiación y de control público de las mismas. 
El primer aspecto básico distintivo de las Administraciones Publicas, según el precitado Informe-Resumen, sería la orientación fundamentalmente no mercantil o lucrativa de éstas en su actividad, que consistiría a su vez en desarrollar dos tipos de actividades: a) la prestación de bienes y servicios, financiados con cargo a impuestos; y b) la redistribución en la realización de actividades no lucrativas, y que no se presten por el sector privado debido a su no rentabilidad mercantil.

El segundo criterio básico caracterizador hoy, conforme al antedicho Informe-Resumen, de las Administraciones Públicas, sería la noción de financiación y control de las mismas, si bien éste se establece de forma diferente de cómo era entendido anteriormente, ya que se va a hacer hincapié más en sus objetivos que en los medios utilizados. El control se entiende hoy como la capacidad decisoria que tienen las Administraciones Públicas y su incidencia social.

Como consecuencia de los dos criterios básicos señalados como hoy delimitadores del concepto de Administraciones Publicas o si se quiere del sector público en un sentido amplio, éstas se subdividirían y se compondrían de tres clases o tipos-, a saber: 1) Las llamadas Administraciones Territoriales (de ámbito central, federal o regional-autonómico en nuestro sistema- y local) y de las llamadas por nuestra doctrina "Administraciones Mediales" al servicio de los órganos constitucionales y las "Administraciones Independientes"; 2) las Entidades Gestoras de la Seguridad Social, en un sentido amplio abarcando a las distintas modalidades institucionales de titularidad pública según cada país; y 3) aquellas instituciones sin un fin lucrativo mercantil controladas y financiadas mayoritaria o totalmente con fondos públicos, siendo un cajón de sastre residual de las anteriores, y comprendiendo a su vez varias clases posibles de organismos públicos.

\section{III) Las descripciones metodológi- cas de las fuentes estadisticas nacionales}

La segunda parte de esta obra incluye la información detallada derivada de la encuesta enviada en 1992 a cada país, con mención expresa de las fuentes, de los organismos intervinientes, del ámbito, de la periodicidad, de la metodología utilizada, del período de referencia de las variables utilizadas, de las clasificaciones utilizadas y de la documentación empleada de veintidós países de la O.C.D.E.

En concreto, las páginas 84 a 86 están referidas a España, utilizándose la fuente de "la encuesta sobre la población activa" elaborada por el Instituto Nacional de Estadística, incluyendo todo el territorio nacional y la globalidad del sector publico, salvo las empresas públicas militares, con una cifra total de empleo público en 1992 de 2.384 .000 personas.

\section{IV) Epilogo}

Esta obra estimo que es de utilidad, en primer lugar, por la información que se aporta de forma bastante detallada sobre la distinta realidad del empleo en el sector público de los países miembros de la O.C.D.E.

En segundo término, pienso que además viene a reafirmar, por la vía de los datos, las dificultades existentes hoy día en profundizar en el análisis comparado del sector público; tarea ésta por otro lado no imposible, si no se olvida, simplificando en exceso, las distintas peculiaridades, antecedentes históricos, y contingencias que existen en cada país. En todo caso, la propuesta de una delimitaciòn de la definición y del contenido del término sector público, y dentro de él de Administraciones Públicas, parece como algo obvio y necesario como punto de partida para la normalización, y tras ésta para el análisis administrativo comparado, permitiendo así extraer posteriormente posibles conclusiones de carácter general, fundadas a su vez en datos fiables y normalizados.

Por último, y para concluir, quisiera señalar que se resalta también en esta obra otro hecho fundamental en la definición, caracterización y contenido del término Administraciones Públicas, que es su carácter sustancialmente no mercantil o lucrativo, o lo que es lo mismo lo ya señalado inequívocamente por nuestra doctrina más solvente como "la especificidad" de las Administraciones Públicas frente a los intentos interesados y/o infundados de equipararlas lisa y llanamente con las organizaciones empresariales de finalidad y carácter fundamentalmente mercantil. Cuestión distinta es la utilización y la adaptación de técnicas útiles de éstas por aquéllas, no debiéndose confundir, por tanto, los meros instrumentos útiles y posibles a utilizar por las instituciones administrativas con la esencia, finalidades y características propias de las mismas.

José-Manuel CANALES ALIENDE Universidad Complutense

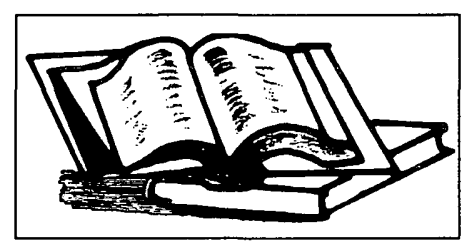

\section{Les fonctions publiques dans l'Europe des Douze}

BODIGUEL, Jean Luc. Librairie Générale de Droit et de Jurisprudence; París, 1994. 177 páginas.

\section{I) Finalidad y planteamiento general de la obra}

La presente obra es ante todo un Vademecum o Repertorio, si bien sintético, que pretende analizar de forma descriptiva la realidad de la función pública de cada uno de los doce países miembros de la Comunidad Europea, fruto de esa penúltima ampliación en la que se incorporaron Portugal y España. Junto a lo anterior, se incluye también al final de la misma un capítulo dedicado a la función pública comunitaria.

La realidad de la función pública no es por supuesto semejante en todos los países, y su conocimiento implica para el autor el estudio no sólo del estatuto jurídico aplicable, sino también la considera- 
ción de la historia, la cultura, las tradiciones, el entorno y los modos de actuación existentes en cada país.

La intención del autor es, sobre todo, a partir del conocimiento de las distintas realidades nacionales que engloban a un total nada despreciable de veintiséis millones de personas empleadas en el sector público de los diferentes países, contribuir a una mejor comprensión mutua y colaborar al esfuerzo común de la construcción de la hoy Unión Europea.

Para realizar este estudio, su autor confiesa en la introducción que ha tenido notables dificultades para poder obtener toda la amplia y diferente información y documentación (normativa, estadística, etc.) necesaria suficientemente actualizada de la totalidad de países, unido a veces a la dificultad de la comprensión de algunas lenguas. No obstante lo anterior, una importante y valiosa documentación obtenida lo ha sido a través de la participación personal del autor en varios Grupos de Investigación Internacionales, así entre otros: el Grupo de Investigación sobre Función Pública Comparada en Europa del Este y del Oeste, el Grupo Europeo de Administración Pública (G.E.A.P./I.I.S.A.), los Encuentros Europeos de la Función Pública (R.E.F.P.),la Asociación Internacional de la Función Pública (A.I.F.P.),y el Instituto Internacional de Ciencias Administrativas (I.I.S.A.).

\section{II) El Autor}

Jean Luc BODIGUEL, autor de la presente obra, es un conocido y prestigioso especialista en temas de función pública, que en la actualidad dirige el Centro de Investigaciones Administrativas de la Fundación Nacional de Ciencias Políticas en París, a la vez que es miembro de numerosos grupos internacionales de expertos en esta materia.

Entre su bibliografía destaca Les anciens élèves de l'Ecole Nationale d'Administration Publique (P.U.F., París, 1978); siendo coautor con Jean-Louis QUERMONNE de La baute fonction publique sous le $V^{\underline{a}}$ Republique (P.U.F., París 1983); y con Luc ROUBAN de Les magistrats, un corps sans âme? (P.U.F., Paris 1991) y Le fonctionnaire détrôné? L'Etat au risque de la modernisation (Presses de la F.N.S.P., París 1971).

\section{III) Analisis del contenido de la obra}

La obra se compone de un total de trece apartados o capítulos, que se corresponden respectivamente y por orden alfabético con cada uno de los doce países miembros de la entonces Comunidad Europea, junto a un último relativo como antes se ha dicho a la función pública europea, con una breve y previa introducción esclarecedora de la finalidad de la obra, acompañada finalmente con una sucinta y básica bibliografía de carácter general, que completa la relativa a cada país que figura a pie de página de los distintos capítulos o apartados del texto.

El primer mérito y aportación de este estudio es la inclusión, frente a la laguna existente hasta la fecha, de un estudio completo que abarca a todos y cada uno de los sistemas de función pública de los doce países, además de la mención de la función pública comunitaria. Existen estudios de carácter parcial y comparado sobre uno o varios países, pero no de todos.

El segundo objeto y aportación del libro es que más que entrar en los pequeños detalles y anécdotas, subraya y pone de manifiesto las principales notas significativas y representativas de la función pública de cada país, poniendo un énfasis especial en la mención de las características del personal al servicio de las Administraciones Territoriales no estatales, tanto de ámbito supralocal (Landers, Regiones, Comunidades Autónomas, etc., según cada sistema político-administrativo) como locales.

El tercer y último gran objetivo y aportación del estudio es el análisis de los distintos aspectos y perspectivas que conforman la realidad plural de cada sistema nacional de función pública, teniendo en cuenta, además de la normativa reguladora, la cultura propia del sistema, procediendo a la consideración de, entre otros, las siguientes características fundamentales de la realidad de un sistema de función pública: datos estadísticos de los efectivos y de su distribución, modelo y configuración territorial de las Administraciones
Públicas, peculiaridades de la gestión, relaciones entre Administración y Política, realidad y praxis sindical, categorías existentes, procedimientos de selección, carrera y formación, derechos y obligaciones, movilidad, sistema retributivo, etc.

Del estudio realizado de las distintas realidades nacionales y de la función pública europea, se extraen unas conclusiones de gran interés aunque no están expresamente explicitadas con carácter general (sin perjuicio de que al final de cada apartado se incluyen con una breve extensión unas conclusiones referentes a cada país).

La función pública de los distintos países de la hoy Unión Europea, configurada mayoritariamente con un sistema de carrera derivado de un Estatuto aprobado con un mayor o menor rango normativo, se enfrenta básicamente con un mayor o menor énfasis y dificultades, en función de la distinta tradición y cultura de cada país, a la búsqueda de una menor rigidez en su funcionamiento. Las experiencias hasta ahora habidas, de distinto contenido y características entre las que se podrían citar las siguientes: contratación del personal procedente del sector privado, desregulación y negociación colectiva de las condiciones de trabajo, mayor movilidad horizontal y vertical, retribuciones complementarias en función de los resultados conseguidos, introducción de una nueva cultura de la gestión pública, sobrevaloración de la formación y de las habilidades en relación a la antigüedad, reforzamiento del poder de los directivos, etc.- no son del todo satisfactorias; no habiéndose resuelto muchos de los problemas existentes y creándose nuevos, en particular, la politización y el clientelismo.

A pesar de lo mucho realizado e intentado, parece que procede un período de reflexión profundo para intentar buscar vías y técnicas que consigan el difícil equilibrio y complementariedad entre la legitimidad y racionalidad legal con la gerencial; o lo que es lo mismo, entre los logros históricos de la tradición y de las garantías del Estado de Derecho con las ineludibles demandas y necesidades ciudadanas actuales, de la eficacia y de la eficiencia. 
Dentro del último apartado o capítulo, dedicado a la función pública europea, merecen especial interés, en mi opinión, además de la descripción general que se hace de sus características y configuración, algunas de las consideraciones que se encuentran dentro de la conclusión final: la dificultad del funcionamiento de la Administración Europea, compuesta por personas procedentes de países con diferentes culturas y lenguas en donde la integración es una tarea muy difícil, unida a la lentitud de los procedimientos administrativos y a la complejidad de la toma de decisiones, junto al difícil equilibrio de los intereses nacionales con los europeos.

\section{IV) Epilogo}

La obra precedentemente reseñada pienso que representa una meritoria y completa aportación, si bien sintética como se ha dicho inicialmente, para el conocimiento de la realidad de la función pública de los doce países de la entonces Comunidad Europea y de la propia función pública comunitaria. Este repertorio deberá, sin duda, en el futuro completarse y enriquecerse con la inclusión del estudio de los países incorporados a la Unión Europea en la última ampliación.

No obstante su indudable interés y actualidad por la visión aportada de cada sistema nacional, que permite fácilmente al lector extraer unas conclusiones y observar una problemática y tendencias comunes, creo que la finalidad expositora de la obra podría haberse enriquecido aún más, con unas conclusiones y opiniones finales del autor sobre el conjunto, plural y complejo, sin duda, analizado. Para concluir, quisiera señalar también que del análisis de las distintas realidades nacionales de la función pública no se extraen ciertamente soluciones de validez y de aplicación universal, pero aún así, por el contrario, estimo que sí se pueden obtener algunas enseñanzas de los éxitos conseguidos y de las errores acontecidos, que pueden ser de utilidad a la hora de acometer con una suficiente reflexión, racionalidad y oportunidad, las modificaciones e innovaciones de la función pública de cada país. Sólo esto, de por sí, justifica suficientemente el interés de la lectura de esta obra.

\section{José-Manuel CANALES ALIENDE}

Universidad Complutense

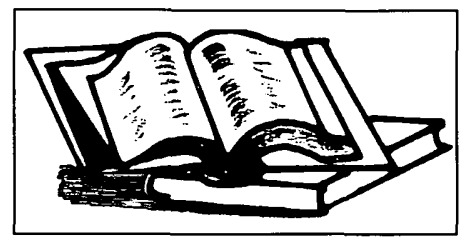

"Les tendances d'évolution des remunerations dans le secteur publique: étude sur neuf pays de l'OCDE 1985-1990"

OCDE. Etudes hors série sur la gestion publique, 1994, número 1; París, 132 páginas.

\section{I) Introducción}

Una preocupación común al conjunto de los países de la OCDE desde los años ochenta ha sido la limitación del crecimiento de los gastos públicos, debido a los persistentes problemas derivados del volumen de los mismos y de los déficit públicos. El período actual ha relanzado el debate por un doble motivo. Por una parte, los países europeos se encuentran implicados actualmente en la construcción de la Unión Económica y Monetaria, habiendo definido conjuntamente planes de convergencia e inscrito en sus programas económicos y monetarios la limitación del crecimiento de los gastos públicos como un objetivo prioritario. Por otra, algunos países miembros se han replanteado recientemente el papel de regulación social y económica que puede y debe jugar el Estado, subrayando en este contexto el problema de la indispensable adaptación del sector público a la coyuntura nacional e internacional. Así, las formas de gestión pública, el volumen de los efectivos del sector público y sus retribuciones se encuentran en el núcleo de los debates.
Los decisores políticos han tomado conciencia de que las retribuciones representan una herramienta de gestión de los recursos humanos, tanto en el sector público como en el privado, y al mismo tiempo que los esfuerzos por tornar más receptivos y flexibles los mercados de trabajo incitan a la puesta en marcha de reformas en ambos sectores, la necesidad de mejorar la eficacia y eficiencia del primero conduce a un conjunto de transformaciones, tanto en los sistemas de retribuciones como en la estructura de las relaciones profesionales. Además, los poderes públicos, al tiempo que deben hacer frente a una limitación de la progresión de los gastos de sus asalariados, están obligados a velar para asegurar los grandes equilibrios macroeconómicos para no crear disfunciones en el nivel microeconómico, por ejemplo, en el nivel de un sector, de una región o de una profesión.

En este contexto, el Comité de Gestión Pública de la OCDE emprendió en 1992 y 1993 un estudio piloto sobre las tendencias evolutivas de las retribuciones en nueve países miembros (Alemania, Australia, Canadá, Dinamarca, España, Finlandia, Francia, Italia y Reino Unido), preparado por Nicole Lanfranchi, del Servicio de Gestión Pública de la OCDE, con la colaboración de expertos oficiales de las diferentes Administraciones nacionales implicadas en el estudio y sobre la base de la información estadística aportada por éstas, con la participación de los Profesores Robert ElliotT, Claudio LuCIFora y Dominique Meurs.

\section{II) Naturaleza, objetivos y estructu- ra del estudio}

Se trata, en efecto, de un estudio piloto, cuyo objetivo esencial consiste en acreditar la factibilidad de una encuesta estadística comparativa de las tendencias en materia de retribuciones, proponiéndose analizar los cambios que ven la luz actualmente en los sistemas de determinación de las mismas en el sector público. La aproximación estadística se completa con informaciones sobre las modificaciones producidas en las formas de determinación de las retribuciones y otros ele- 
mentos de naturaleza cualitativa. Pero conviene recalcar que se trata de una primera investigación y no de un examen completo de los procesos que operan en el sector público. Por lo tanto, en esta fase "preliminar" el Comité de Gestión Pública de la OCDE no se ha propuesto realizar una descripción detallada de las tendencias evolutivas de las retribuciones, ni deducir conclusiones definitivas de los datos observados. Por el contrario, el estudio, presidido por una gran honestidad en la exposición de sus limitaciones y condicionamientos, reconoce expresamente que debe ponerse la mayor precaución en el uso e interpretación de sus datos, así como la existencia de un conjunto de problemas metodológicos no resueltos aún, en particular los vinculados a la definición del campo del sector público. Por otra parte, debe tenerse en cuenta permanentemente el contexto social y económico de los países en los que se inscriben los datos obtenidos. En definitiva, el informe se concibe, esencialmente, como un ejemplo del tipo de análisis que es posible realizar en este terreno, y no como un intento de obtención de resultados.

El estudio ha recurrido a dos tipos de análisis basados, el primero, en los datos agregados del sector público y de las variables macroeconómicas, y el segundo, en los datos individuales sobre las retribuciones de un asalariado tipo de una muestra de profesiones. Doble aproximación que responde al deseo de estudiar las modalidades efectivas de paso entre dos lugares privilegiados del proceso de determinación de las retribuciones: por un lado, el de la elaboración de las grandes orientaciones y de la toma de decisiones políticas que se traducen en última instancia en la adopción del presupuesto del Estado; y por otro, el más o menos descentralizado según los países de la determinación individual efectiva de las retribuciones. Así se pretende tanto identificar las tendencias resultantes de las estrategias salariales de los poderes públi$\cos$, como aprehender nuevas formas de determinación de las retribuciones.

Con tales finalidades el estudio se estructura en una introducción y en tres partes: la primera, dedicada al análisis de los datos agregados, comprendiendo el encuadramiento del sector público en cada economía nacional, el objetivo común de la limitación del incremento de los gastos de personal y la emergencia de nuevas prácticas en la gestión del personal del sector público; la segunda, que salta a la esfera microeconómica, analizando las retribuciones individuales, incluyendo los componentes de tales retribuciones, las lógicas evolutivas en el período 1985-1990 y un análisis comparativo exploratorio de las mismas; la tercera, finalmente, comprende las conclusiones. El estudio se completa con cuatro detallados Anexos, comprendiendo la lista de los expertos nacionales designados y las fuentes usadas en cada país para la recolección de los datos agregados (Anexo 1); las principales características de los sistemas de determinación de las retribuciones del sector público en cada país considerado (Anexo 2); una nota metodológica explicando la selección de las profesiones elegidas y el método seguido para la elaboración de la encuesta sobre las retribuciones individuales (Anexo 3); y tres breves resúmenes sobre los sistemas retributivos del sector público en Dinamarca, España y Reino Unido, junto con una nota referente a la definición de dicho sector en Canadá, país que sirve de ejemplo sobre la posible diversidad en la delimitación del mismo y los problemas metodológicos que presenta tal tarea (Anexo 4).

\section{III) Análisis del contenido de la obra}

El estudio se propone, en primer lugar, obtener una visión comparativa del lugar del sector público en el seno de la economía nacional a comienzos de los años 90. Las retribuciones del sector público, por su volumen, constituyen un factor que va a influir más o menos fuertemente en los grandes equilibrios macroeconómicos, actuando sobre la inflación y sobre el nivel de empleo. Sin embargo, no todos los países afrontaron en el mismo momento el problema de la inflación, encontrándose en etapas distintas al comienzo de la década. La comparación de la evolución de los salarios reales de los sectores públicos refleja las grandes elecciones políticas nacionales.
Así, mientras que el deseo de yugular la inflación ha conducido a algunos países a imponer a los asalariados del sector público una baja en su poder de compra, en otros la aplicación de una política de rigor salarial se ha realizado desde el respeto al principio de mantenimiento de la capacidad de compra.

A partir de la recolección de los datos estadísticos, el informe destaca la detección de variaciones en los efectivos y de los costes salariales, permitiendo formular hipótesis sobre la emergencia de nuevas prácticas en la gestión de los recursos humanos en el sector público. Así, en algunos países los equilibrios entre los gobiernos centrales y los locales se han modificado en favor de los últimos, pero la descentralización en la determinación de las retribuciones no significa por ello sinónimo de economías, pudiendo acompañarse de una deriva salarial difícilmente controlable. En otros países, las modificaciones en reparto de los efectivos y los gastos aparecen en el nivel de los subsectores funcionales, reflejando la necesidad de tener en cuenta tensiones existentes en el mercado de trabajo. E incluso puede aparecer una modificación sustancial de los efectivos si se tiene en cuenta el estatuto del empleo, siendo la idea subyacente la de que la remuneración de los agentes contratados presenta un grado de flexibilidad superior al de los funcionarios. En definitiva, toda tentativa de aproximación a la evolución de los efectivos y de los costes salariales debe realizarse considerando las mutaciones que intervienen en la estructura de aquéllos.

El estudio resalta que tampoco las modalidades de evolución de la masa salarial revisten los mismos aspectos en todos los países, dependiendo de condiciones económicas y sociales particulares de cada uno de ellos, entre los que destacan el sistema de relaciones profesionales y la eventual búsqueda de un consenso social. Así, mientras en unos países el fundamento de la política salarial consiste en la actuación sobre la evolución de las retribuciones de los agentes del sector público para contener la masa salarial, en otros se concede más importancia a los mecanismos de fijación de las retribucio- 
nes y al resultado final que a una anticipación del volumen de la masa salarial.

La parte segunda del informe intenta obtener, mediante la observación de las transformaciones de los componentes de la retribución individual, una aproximación satisfactoria a las nuevas prácticas retributivas. Y los resultados de la encuesta acreditan que es posible hacer variar las retribuciones individuales en el sector público, sea en función de las características del agente, sea en función de un grupo profesional determinado, con independencia de las medidas de incremento generalizado. Las modificaciones de los sistemas salariales inicialmente puestas en marcha responden a la necesidad de tener en cuenta nuevas cualificaciones y especificidades profesionales, competencias particulares, el rendimiento o la productividad de los agentes e incluso déficits en el mercado de empleo local o profesional. Las respuestas técnicas son variadas. Así, se ha acudido en algunos Estados a las primas individuales, que sin embargo encuentran a veces dificultades de aplicación. En cambio, la refundición total o parcial del sistema de clasificación de los funcionarios asegura una nueva consideración de las cualificaciones y nuevas posibilidades de carrera, permitiendo a la vez introducir factores de diferenciación en la fijación de las retribuciones individuales. Por otra parte, se marca una clara tendencia a asociar la progresión de los salarios al rendimiento personal más que a la antigüedad.

Evidentemente, las distintas opciones en materia de gestión de los empleos y de determinación de los salarios individuales no son neutrales en relación con el presupuesto. Así, un sistema de carrera que garantice una progresión salarial vinculada a la antigüedad supone una rigidez a la baja de la masa salarial, que se acentuará en períodos de reducción de nuevas selecciones y de envejecimiento de la población empleada. En un sistema que privilegie el contenido de los puestos y no las características del individuo, habrá una mejor adecuación entre el volumen de la masa salarial y las definiciones de los puestos, pero las medidas de reclasificación que tal sistema comporta -normal- mente introducidas en un contexto de modernización del sector público- entrañan costes presupuestarios importantes.

\section{IV) Epilogo}

En conclusión, puede señalarse que el período de observación considerado en la encuesta piloto es aún demasiado breve para permitir deducir en todos sus efectos las nuevas políticas que se ponen en práctica progresivamente. No obstante, la metodología empleada ha demostrado que es técnicamente posible obtener tendencias significativas, y que es necesario completar el análisis cuantitativo con informaciones de naturaleza cualitativa. Las experiencias de los distintos países analizados demuestran claramente que el peso de las costumbres en el sector público puede constituir un obstáculo para el logro de los objetivos fijados por las instancias políticas, y que el éxito de cualquier política salarial pasa por el reconocimiento por parte de todos de la necesidad de integrar una nueva dimensión, que es la de la gestión del personal del sector público. En esta búsqueda de una reducción de sus costes salariales y de políticas retributivas innovadoras,se puede obtener provecho de las informaciones obtenidas de las diversas experiencias nacionales.

Cabe agradecer especialmente a sus autores su rigor técnico, claridad expositiva -apoyada en un abundante y bien estructurado aparato gráfico- y la honestidad con que exponen tanto las posibilidades como las limitaciones metodológicas del estudio realizado. De su capacidad de comprensión de las situaciones nacionales y de la fiabilidad de las fuentes usadas pueden servir de muestra, fácilmente cotejable por nosotros, los comentarios y exposiciones que se contienen en relación con España. En pocos párrafos, y con una imparcialidad aséptica pero lúcida, efectúan un excelente esbozo del panorama que ofrece nuestro país en materia de gestión de recursos humanos y política retributiva en el sector público.

José-Manuel RODRÍGUEZ ALVAREZ Universidad Autónoma de Madrid

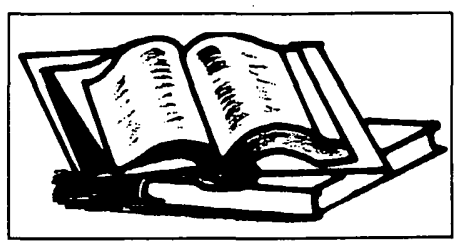

"La rémunération des hauts fonctionnaires: étude sur onze pays de l'OCDE 1980-1991"

OCDE. Etudes hors série sur la gestion publique, 1994, número 4; París, 70 páginas.

\section{I) Introducción}

El estudio objeto de esta recensión preparado por el Profesor Christopher Hood y Sonia LamberT, de la "London School of Economics", y María MAGUIRE, del Servicio de Gestión Pública de la OCDE, sobre la base de los datos aportados por las autoridades nacionales de los países seleccionados-, tiene por finalidad el análisis de los niveles retributivos de la alta función pública y su evolución entre 1980 y 1991 en once países miembros de la citada organización: Alemania, Australia, Canadá, Estados Unidos, Finlandia, Francia, Irlanda, Japón, Países Bajos, Reino Unido y Suecia.

Ciertamente las retribuciones de los altos funcionarios representan tan sólo una parte ínfima de los gastos totales del Estado, e incluso en una situación caracterizada por las restricciones presupuestarias y un recorte sustancial de los gastos un aumento sustancial de las mismas no produciría un efecto notable sobre el gasto público total. Sin embargo, la cuestión presenta un interés muy superior al que se deriva de tal consideración meramente cuantitativa, revistiendo una importancia crítica para la gestión pública la forma en que se retribuye a los altos funcionarios por tres razones: contribuye a determinar el perfil y las motivaciones de las personas que se sienten atraídas por el servicio público, influye en la manera en que los altos funcionarios cumplen sus tareas y, por último, el nivel y la estructura de sus retribuciones constituyen indica- 
dores importantes para los demás asalariados del sector público y, en general, para el conjunto de la población activa.

\section{II) Objetivos y estructura del es- tudio}

El estudio examina, fundamentalmente, las diferencias existentes entre los niveles de remuneración y las estructuras retributivas de los altos funcionarios en esos países -entendiendo por tales los pertenecientes a los tres niveles más elevados de la alta administración de cada uno de ellos-; la evolución del poder de compra -expresado en paridades de poder de compra convertidas en dólares norteamericanos- de los ingresos percibidos por la alta administración en el período indicado; la evolución de la distancia entre la retribución de los altos funcionarios y las de los agentes de la función pública pertenecientes a las categorías inferiores que sirven de "vivero" para el reclutamiento del personal de dirección; la comparación con las evoluciones de las retribuciones en el sector privado, del crecimiento económico y del PIB per capita; las fórmulas institucionales de determinación de las retribuciones de los altos funcionarios y la evolución de estos sistemas.

Con tal finalidad, el informe se estructura en cuatro partes: la primera (Introducción) efectúa una sucinta referencia a la problemática presentada por las retribuciones de estos funcionarios, incluyendo el plan, campo y límites del estudio; la segunda se refiere al nivel, estructura y tendencias de la retribución de los altos funcionarios; la tercera analiza los procesos de determinación de tales retribuciones (comisiones de examen, negociación individualizada, negociación colectiva y vinculación con el resto del servicio público); y la cuarta, finalmente, incluye las conclusiones y las cuestiones fundamentales de fondo. El informe se completa con siete oportunos Anexos, referentes a los grados y niveles de la función pública considerados en el estudio (Anexo A); la proporción en cada país de altos funcionarios en relación con la población activa no agrícola (Anexo B); un resumen de los sistemas de retribución de los altos funcionarios en cada país (Anexo C); las distancias relativas de los salarios en el seno de la alta función pública en el período considerado (Anexo D); las distancias relativas de los salarios entre la alta administración y los niveles inferiores que sirven de "vivero" de selección para la misma (Anexo E); la evolución porcentual del salario en el nivel más elevado de la función pública, del salario del obrero medio y del PIB por habitante en el periodo indicado (Anexo F); y los métodos institucionales de determinación de los salarios de los altos funcionarios en los países objeto de estudio (Anexo $G$ ).

El estudio, no obstante, no se refiere a todas las remuneraciones que integran la retribución de los altos funcionarios, limitándose al salario base, salvo en aquellos casos (Alemania, Japón) en que primas e indemnizaciones sustanciales se integran automáticamente en la retribución. Tampoco aborda las ventajas o prestaciones no retributivas, tales como las pensiones de jubilación y los seguros de enfermedad, así como otros factores referentes a la retribución durante todo el ciclo de vida activa, como las indemnizaciones susceptibles de ser percibidas con ocasión de la salida de la función pública, o el conjunto de ingresos percibidos en el curso de toda la carrera pública y las posibilidades de empleo en los sectores público o privado que se ofertan a los altos funcionarios.

\section{III) Análisis del contenido del es- tudio}

El estudio pone de manifiesto que los niveles del salario base de los altos funcionarios varían considerablemente en los países considerados, si bien las comparaciones interestatales deben ser interpretadas con suma precaución, al ser numerosos los factores que inciden en tal circunstancia. Por ejemplo, las diferencias pueden derivarse en cierta medida de las posibilidades que se ofrezcan a los altos funcionarios de ocupar puestos bien remunerados en el sector privado después de haber abandonado el servicio público, la dimensión de la función pública del Estado en cuestión, o incluso la importancia de la capital en que trabajan (si ésta es un destacado centro financiero y de negocios, como es el caso de Londres o Tokio).
También varía la estructura de las retribuciones en los diferentes países analizados, explorando el informe, preferentemente, tres aspectos: la medida en que la determinación de las retribuciones de los altos funcionarios se realiza de manera diferente a la de los escalones inferiores de la función pública; la medida en que la retribución es fijada atendiendo al grado o rango del trabajo realizado, o a una evaluación de los rendimientos individuales; y la medida en que la retribución global comprende primas e indemnizaciones sustanciales o se compone principalmente del salario base.

Asimismo el informe pone de relieve que se producen evoluciones muy diferentes en los niveles de las retribuciones. Mientras en cuatro de los países analizados se ha producido una disminución del poder de compra de los altos funcionarios en términos reales, en otros tres los ingresos han aumentado muy apreciablemente en términos reales. Y si algunas de estas evoluciones han sido determinadas por diferentes tasas de crecimiento del PIB por habitante, también hay otros factores que pueden incidir en las mismas, como es el caso, hasta cierto punto, de los diferentes sistemas institucionales usados para la determinación de las retribuciones.

La remuneración de los altos funcionarios se encuentra determinada por negociaciones individualizadas en cuatro de los países considerados, al menos parcialmente. Cinco países aplican un elemento de remuneración vinculado al rendimiento para algunas o la totalidad de sus altos funcionarios. Y un acuerdo colectivo determina las retribuciones en al menos la mitad de los países analizados. En tres de ellos, las negociaciones con los sindicatos constituyen un importante elemento en proceso decisorio. En cuatro países el salario de los altos funcionarios se encuentra directamente vinculado a las retribuciones del resto de la función pública; y en cuatro también está determinado por las comisiones de examen de las retribuciones. Una de las conclusiones más sorprendentes del estudio en este aspecto radica en el hecho de que los países que han retribuido más generosamente a los altos funcionarios no son los que han recurrido de manera significativa a las negociaciones individualiza- 
das, sino las que se han basado en una comisión especial o al establecimiento de una vinculación con las retribuciones de los niveles inferiores de la función pública. En cambio, sorprende menos que los países que se sitúan en la parte inferior de la horquilla sean aquellos que tradicionalmente recurren a la negociación colectiva para determinar las retribuciones de los altos funcionarios. Pero más importante aún es la conclusión de que, bajo los efectos de factores más generales, el mismo procedimiento de determinación puede producir resultados retributivos muy diferentes, y que en este terreno más importante que las reglas institucionales es el clima político general.

Tras la evidencia de la inexistencia de uniformidad o de convergencia en lo que se refiere a las retribuciones de los altos funcionarios en los once países de la OCDE considerados, el estudio examina la dimensión política de las mismas adoptando una posición fundada en el análisis utilitarista clásico elaborado por Jeremías BENTHAM en su conocida obra The Rationale of Reward, publicada en 1825, a su vez influida notablemente por el pensamiento de Adam Smith. Tal posición se expresa en diversos principios: las recompensas del servicio público deberían concebirse de manera que el deber se corresponda con el interés personal; deben dirigirse a maximizar la calidad del servicio, a limitar los salarios y a igualar las retribuciones nominales y reales. Además, los gastos derivados de un empleo público deberían ser asumidos por la comunidad de beneficiarios del servicio; los niveles de las retribuciones deberían vincularse al grado de "confianza" o de "tentación" que implica un empleo público; y los funcionarios deberían beneficiarse de "pensiones de jubilación" adecuadas. Para los autores del estudio, estos viejos principios mantienen toda su pertinencia en la actualidad, debiendo ser todo sistema de retribución de los altos funcionarios el producto de un compromiso entre estos principios en cierto modo antagónicos.

\section{IV) Epílogo}

Para concluir, cabe resaltar que del estudio se deduce claramente la inexis- tencia de un método único para satisfacer estos objetivos de la política de retribución de los altos funcionarios, del mismo modo que no hay una "retribución global" estandarizada a escala internacional para los mismos. De hecho, la principal utilidad de las comparaciones internacionales referidas al sector público consiste en permitir el estudio conjunto de las posibilidades que se ofrecen, complementando así las clásicas comparaciones realizadas en los diferentes países entre el sector público y el privado, gracias a las informaciones suplementarias suministradas por aquéllas.

Y es posiblemente en la misma inauguración de los estudios comparativos interestatales en el ámbito del sector público donde radica el mayor interés del informe, abriendo con ello un campo de análisis y trabajo sugerente y delicado, al tiempo que contribuye a "desmitificar" y a acabar con tópicos en una materia donde siempre han sido fáciles las demagogias interesadas.

José-Manuel RODRíGUEZ ALVAREZ

Universidad Autónoma de Madrid

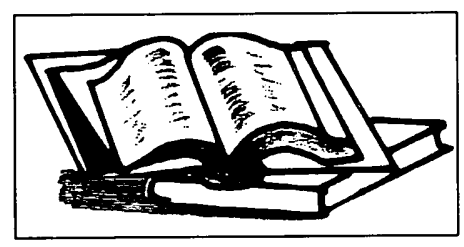

\section{La negociación colectiva de los funcionarios públicos}

ARENILLA SAEZ, Manuel. Editorial La Ley.

La resistencia a emplear los nuevos enfoques para el estudio de la gestión pública es moneda común en la mayoría de los trabajos relativos a la variada temática sobre Función Pública, la cual, siendo objeto de estudio hasta ahora por una densa y prolífica literatura producida desde el ángulo jurídico-administrativo, no ha conseguido, por el contrario, y hasta tiempos recientes, la atención de los sectores académicos y profesionales vinculados al moderno paradigma que arranca en la visión multidisciplinar inherente a la ya consolidada Ciencia de la Administración.

No es éste el caso de la obra que ahora se comenta, que viene a configurarse como el trabajo de mayor importancia sobre esta materia de los realizados hasta el momento presente, así como referencia insustituible para un completo entendimiento y captación del problema de la Función Pública en la dimensión de las relaciones colectivas de empleo, al ser precisamente el tratamiento dado por el autor al tema estudiado el elemento principal para ello.

Por otro lado, si la proyección al análisis realizado por el profesor ARENILLA del método propio de la disciplina que imparte en la Universidad de Granada singulariza indudablemente su obra, no es menos atrayente para el lector el hecho de que ésta se vea enriquecida por su experiencia como gestor público en la materia tratada, circunstancia que atraviesa el libro de principio a fin, incluso en sus partes más próximas al enfoque politológico.

La obra se divide en tres partes que cumplen, a su vez, como señala el propio autor, tres funciones distintas, siendo precedidas por una introducción en la que se centran prodigiosamente los fenómenos esenciales que conforman la realidad y las dificultades por las que atraviesa la Función Pública española, sus riesgos e incertidumbres, en una perspectiva que atiende al marco polidimensional sobre el que necesariamente deben sustentarse las claves explicativas para el conocimiento de la acción sindical y la contratación colectiva en el empleo público.

En la primera parte el profesor ARENILLA se arriesga a sugerir una nueva forma de ver la Administración, según la cual la conexión entre la Administración y el poder político, desde la instrumentalidad de aquella sobre éste para buscar la fuente de su legitimidad, es tanto más problemática si se le añaden otras notas singulares propias de nuestro tiempo, 
como son el problema de la eficiencia administrativa y de la publificación de intereses grupales. Como vía para resolverlo precisa que aunque la instrumentalidad continúa suministrando un elemento conceptual imprescindible, hay que sumar para su estudio como objeto específico de conocimiento diversas "lentes conceptuales".

Plantea de este modo la necesidad de utilizar el enfoque de la Ciencia de la Administración para explicar estos fenómenos, sin perder de vista la ubicación de la Administración dentro del sistema político, o su imbricación en el Estado, situando también las nuevas preguntas en el contexto de las tensiones PolíticaAdministración, Estado-Administración y Estado-Sociedad.

Rinde un tributo a la teoría institucionalista ( representada principalmente por Chevaller y LoschaK ), expresando sus insuficiencias a la luz de estas nuevas preguntas, para, finalmente, ante la difuminación actual de la idea del Estado, encontrar en la perspectiva de las relaciones entre personas y grupos organizados el medio específico de comprensión de la acción sindical en las organizaciones públicas.

La segunda parte gira sobre las características profundas de la acción sindical en la Administración, más que sobre su marco normativo, que se considera resultado de aquélla. Para el autor, en este orden de cosas, habría aparecido una Administración "presionada", por la falta de costumbre de tratar cuestiones que hasta ese momento estaban dentro de la unilateralidad y por la utilización de técnicas ajenas al principio de "supremacía", existiendo no obstante unos antecedentes históricamente claros del sindicalismo en la Administración.

A pesar de ello, no se olvida el tratamiento de la fundamentación jurídica del modelo, que concreta en el análisis de la regulación constitucional de la Función Pública y de los proyectos de ley anteriores a la Ley 30/84, así como de la Ley Orgánica de Libertad Sindical, de la jurisprudencia constitucional en la materia y de los vigentes instrumentos normativos sobre negociación colectiva.
A su juicio, los intentos de equiparación de la naturaleza de los conceptos de funcionario y trabajador y la aplicación del criterio de irradiación de la mayor representatividad sindical han acarreado consecuencias negativas porque cercenan las posibilidades de integración decisional del conjunto de los actores del "interior" de la Administración, en especial de los funcionarios directivos, obviándose también a los grupos minoritarios, aunque tengan importancia sectorial, frente a los grandes Sindicatos del sector privado.

Asimismo, dibuja una clara descripción de los problemas y del contexto en el que éstos nacieron y se desenvolvieron, en la construcción del modelo normativo de Función Pública, centrándose en las quiebras con las que éste se desarrolla, la improvisación y falta de lealtad institucional que caracterizó a los procesos de transferencias y que tan- decisivamente condicionaron la evolución hacia lo que llama "jerarquía institucional" de la negociación colectiva, la politización, la heterogeneidad de los recursos humanos traspasados y el anticorporativismo, con un pronunciamiento contrario a las tendencias laboralizantes, de las que realiza un examen exhaustivo.

A partir de lo que denomina visión estática de la regulación inicial (la Ley 9/87) el autor señala al estrangulamiento de la capacidad normativa de las Comunidades Autónomas y a las disfunciones del sistema de representación elegido, que no sería más que una forma de recuento de votos para avalar la preeminencia de los Sindicatos más representativos en los "foros superiores": Consejo Superior de la Función Pública y Mesa General de Negociación, como principales rasgos del modelo, calificando como reflujo derivado del cierto temor que se tenía a las consecuencias de la extensión de la participación sindical, el dato de que no se contemple expresamente en la Ley 9/87 el derecho a la negociación colectiva.

Dedica aquí una profunda reflexión a las implicaciones que presenta la negociación en la Administración: "politización", "cambio de régimen de los burócratas", etc., y una crítica a la distinción legal entre Acuerdos y Pactos, por la falta de alcance real que tiene esta segunda categoría.
La ampliación del concepto de "negociación" derivada de la Ley $7 / 89$ refuerza para el autor el papel preeminente de los Sindicatos más representativos en la negociación colectiva, consolidando el modelo que trae causa de la LOLS. También se refuerzan la jerarquía política que opera en la negociación, por el carácter federal de los distintos actores, la tendencia anticorporativa y la configuración de un papel de los Sindicatos más representativos como grupo de presión en el proceso legislativo, referido a una serie de materias que, aun con reserva legal, pasan a ser negociables.

Se ocupa una gran parte de la obra al estudio del Acuerdo Administración - Sindicatos de 1991, explicitando los, a su entender, negativos efectos de la exclusión de organizaciones o grupos con intereses sectoriales no encuadrados en los Sindicatos mayoritarios, indicando, desde otro punto de vista, que el hecho de la marginación del ámbito de aplicación de una parte del Acuerdo a segmentos funcionariales de niveles superiores va en la tendencia de ir generando un estatuto singular para este tipo de personal ("la función directiva"). También subraya la forma de ordenación de la negociación colectiva en base a una serie de principios como uno de los aspectos más destacables del Acuerdo, si bien con una crítica a la estructuración de los nuevos ámbitos descentralizados de negociación, por cuanto no resuelven el vaciamiento de contenido real de los órganos de representación.

En relación con el desarrollo de los Acuerdos del 91, el autor entiende que la importancia del mismo no está tanto en los contenidos del Acuerdo como en los valores que impone: ordena y clarifica la negociación en los diversos ámbitos, aunque se "sacrifica" la representatividad, y consolida la jerarquía negociadora y el deseo de la Administración de negociar con pocos.

La visión dinámica o factor dinámico del sindicalismo lleva al autor hacia un intento de búsqueda de un concepto de interés general, penetrando en las teorías neocorporatistas, con la conclusión de que es la realidad social el factor determinante del interés a proteger y no el Derecho positivo. 
El interés de la Administración vendría determinado por las directrices políticas de sus órganos de Gobierno. Si tradicionalmente los burócratas jugaban un papel relevante en este sentido, hoy el aislamiento de éstos entre la clase política y los trabajadores los sitúa en una posición ambigua, toda vez que el interés de la Administración se forma también por fuerzas provenientes del exterior (los Sindicatos y otros actores), tornándose mucho más flexible. La singularidad del interés sindical quedaría precisada por la contribución al interés general desde su condición de actor político-social, publificándose sus intereses, aunque se mantengan todavía las percepciones derivadas de su tradición histórica reivindicativa y de su papel en el sector privado.

La participación sindical como factor externo la ve como característica derivada de la mayor representatividad, que se expresa como "politización" de las relaciones, influyendo decisivamente este carácter en la formación de los Acuerdos, así como en su mayor eficacia integradora del conflicto, sin perjuicio de las posibles disfunciones para determinados intereses sectoriales, puesto que predomina la necesidad de alcanzar la paz social. Este elemento es a su vez identificativo de las claves de los conflictos o quiebras del sistema, que se apreciarán en términos de incidencia sociopolítica por ambas partes, incidiendo también en la composición de los elementos axiológicos definitorios de los comportamientos en la negociación.

La tercera parte del libro es una proposición para el diseño de nuevos caminos que neutralicen los peligros que el autor ve en el modelo elegido.

Sugiere que la participación, concebida como apertura a nuevos actores sociales junto a los Sindicatos, en el proceso decisional, aun teniendo en cuenta sus dificultades, evitaría los riesgos de la cogestión desde el binomio Administración - Sindicatos mayoritarios, aunque no obstante también ésta tendría, en opinión del autor, algunas ventajas.

Plantea finalmente la obra la posible definición de un papel integrador de la Administración mediante la ocupación por ésta de una posición central en relación con los grupos sociales y el poder político, cumpliendo la misión de cauce comunicativo entre el poder y la sociedad, sosteniéndose que los cambios de la Administración implican una modificación en el sistema político que debe lograrse desde el acuerdo social, por lo que esta alteración de las relaciones entre lo público y lo privado dependería de la existencia de un consenso para una nueva cultura de los servicios públicos y de la sociedad.
Es obligado, por último, significar el reconocimiento a la labor del profesor ARENILLA y a su esfuerzo por dotar de entidad científica al tratamiento de un tema tan complejo como es la negociación colectiva de los funcionarios públicos, lo cual hace que esta obra sin perjuicio de su alto nivel científico sea a la vez de utilidad para la práctica de la gestión, siendo de esperar que este esfuerzo se complemente con nuevos trabajos en los que la utilización de técnicas empíricas, permita aportar mayor solidez a las consideraciones que efectúa acerca de la relatividad del fenómeno sindical en la Administración Pública por la diferente implantación de los agentes sindicales en las que denomina zonas operativa y decisional, o a la discutible crítica, que no compartimos, formulada a la recepción en las organizaciones públicas del criterio de mayor representatividad sindical y su atribución a ésta de la condición determinante de las disfunciones del modelo.

También sería deseable que la continuidad de la línea emprendida por el autor fructificara en una menor compartimentación de los enfoques sobre los distintos aspectos que conforman el objeto estudiado, para evitar que la multidisciplinariedad no devenga finalmente en una pérdida de visión de conjunto integral.

Julián SÁNCHEZ-VIZCAÍNO CASTRO 